\title{
A study on Influence of CCPM+ Parameters on Project Scheduling
}

\author{
Zhang Fan \\ School of Architecture and Engineering, Yan'an University, Yan'an 716000, China \\ 121604203@qq.com
}

Key words: buffer sizing, CCPM+, project scheduling

\begin{abstract}
To tackle problems in traditional project system, CCPM (Critical Chain Project Management) was introduced by Goldratt(1997) to identify both task dependence and resource conflicts as main constraints within a project[1]. In this paper, Critical Chain software CCPM+ is utilized to automate the Critical Chain planning process on an example project, from which more in-depth discussions are made based on computerized simulation process by CCPM+. It includes the investigation on the influence of different parameter values on project duration and buffer sizing.
\end{abstract}

\section{Introduction}

Although CCPM has achieved some success, problems still exist in real project that necessitates further study on its application. Given the fact that software has become an indispensible aid to modern project works, Critical Chain software CCPM+ is hereby used to create a CCPM schedule through data experimental to test result. It is much convenient than the manual one, especially for the large and complex projects forming several critical chains. The use of CCPM+ can contribute to improving the existing Critical Chain capacity.[2].It helps to "simulate weeks, months or even years in seconds of computer time. “, making it convenient for project planning and scheduling.[3]

\section{Case of computerized CCPM simulation}

In order to achieve research purpose, an example adapted from Schieman (2002) has been used. There are two projects taking place simultaneously: one is the kitchen project, the other is the bedroom project. The software would help to create a Critical Chain schedule after following CCPM scheduling procedure, including: 1 . Define tasks and select the MS Project schedule options. 2.Build task network. 3.Resource level the network. 4.Identify the Critical Chain. 5.Insert Feeding and Project Buffers.[4]

After these procedure, CCPM results demonstrate that duration of the project buffer (46.5 days) is estimated as the half of the reduced duration (93 days). The duration of the schedule at this stage is shortened to 150.5 days, compared with the leveled duration of 186 days, which is the result of the traditional critical path method. Also, this method enables the bedroom project to start as late as possible and reduce multitasking.

\section{Data and analysis}

\subsection{How factor applied impacts the project duration}

CCPM+ uses the 50/50 duration estimate as default based on a normal distribution. To explore further the relationship between factor applied and duration of projects, the following statistics are presented though trial of CCPM+ under various factors. It is shown in table 1.1 and figure 1.1 below. The factor in CCPM+ is the ratio of duration and low-risk duration.

As table 1.1 shows, a range of assumptions on factors have been listed, which generated corresponding results of duration of projects. Predictably, the larger the factor (duration/ low-risk duration) is applied, which means that the longer the duration of whole project would be (since the low-risk duration is fixed). It is because an increasing amount of safety concerns have been added when to apply larger amount of factors. 
However, the duration of bedroom project (including project buffer) shows the opposite trend, with a minimal decrease along with the added safety. Nevertheless, the extent of its decrease is much slighter than the increase of trend in kitchen project, so the overall duration shows the increasing trend. To figure out the reasons why the duration of bedroom project (including project buffer) decreases, the project buffer and the duration of bedroom project are listed separately shown in the last two rows of table 1.1 .

As for the reasons why the project buffer duration decreases as with the larger factor (duration/ low-risk duration) applied, it could be explained as follows: the larger factor means the longer "duration" estimated considering increasing amount of safety, since "the low-risk duration" is fixed. When more safety consideration has been taken gradually, project buffer, which manage risks of possible delays, are set with a smaller size. Otherwise, if the project buffer also increases or remains stable, the excessive buffer would be added. It is worth to note that the column in bold with 0.5 applied is the standard practice recommended by scholars. Under this factor, the project buffer (46.5 days) is sized as half of duration (93 days).

Table 1.1 the simulation of how factor applied affects duration of projects

\begin{tabular}{|cccccccc|}
\hline Factor applied(duration/ low-risk duration) & $\mathbf{0 . 3 5}$ & $\mathbf{0 . 4 0}$ & $\mathbf{0 . 4 5}$ & $\mathbf{0 . 5 0}$ & $\mathbf{0 . 5 5}$ & $\mathbf{0 . 6 0}$ & $\mathbf{0 . 6 5}$ \\
$\quad$ & 142.9 & 145.5 & 148 & $\mathbf{1 5 0 . 5}$ & 153.1 & 155.6 & 158.2 \\
Overall duration of project (days) & & & & & & & \\
The duration of kitchen project (days) & 82.4 & 89.6 & 96.8 & $\mathbf{1 0 4}$ & 111.2 & 118.4 & 125.6 \\
$\begin{array}{c}\text { The duration of bedroom project (days) } \\
\text { (including project buffer) }\end{array}$ & 122.9 & 121.4 & 120 & $\mathbf{1 1 8 . 5}$ & 117.1 & 116 & 115.3 \\
$\begin{array}{c}\text { The duration of bedroom project (days) } \\
\text { project buffer(days) }\end{array}$ & 62.45 & 65.6 & 68.8 & $\mathbf{7 2}$ & 75.25 & 78.8 & 82.7 \\
\hline
\end{tabular}

Figure 1.1 the diagram corresponding to various factors

\subsection{Buffer sizing techniques in CCPM+}

Obviously the size of a buffer has a significant impact on the project schedule. According to Advanced Projects (2008), CCPM+ provides two methods of sizing buffers: The Fixed length method and Fixed plus SSQ method [5]. The fixed length method is similar to the Cut and Paste Method while Fixed plus SSQ method is actually similar to the Root Square Error Method (RSEM) mentioned above. Advanced Projects (2008) instructs to set the percentage as zero while using the Fixed + SSQ method and 0.5 for the fixed length method.

The detailed results have been given in the table 1.2. As can be seen from it, the project buffer sized by the $50 \%$ fixed length is larger than the SSQ method under the recommended parameter set in each method. Also, there is a trend that the larger the factor is, the smaller the difference between these methods would be found. Therefore, while adopting these two different buffer sizing techniques, the two ways may cater for different situations. 
Table 1.2 the simulation of two buffer sizing methods in CCPM+

\begin{tabular}{|c|c|c|c|c|c|c|c|}
\hline Factor applied(duration/ low-risk duration) & 0.35 & 0.40 & 0.45 & 0.50 & 0.55 & 0.60 & 0.65 \\
\hline overall duration of project (days) & 142.9 & 145.5 & 148 & 150.5 & 153.1 & 155.6 & 158.2 \\
\hline Project buffer by $50 \%$ fixed length(days) (2) & 60.45 & 55.8 & 51.2 & 46.5 & 41.85 & 37.2 & 32.6 \\
\hline Project buffer by SSQ(days) & 47.2 & 43.6 & 40.0 & 36.3 & 32.7 & 29.1 & 25.42 \\
\hline $\begin{array}{l}\text { The percentage of difference in project duration } \\
((2)-(3)) /(1)\end{array}$ & $9.27 \%$ & $8.38 \%$ & $7.57 \%$ & $6.78 \%$ & $5.98 \%$ & $5.21 \%$ & $4.54 \%$ \\
\hline
\end{tabular}

\section{Conclusion and recommendation}

The result of simulation in this paper indicates the influence of factor applied on the project duration, buffer sizing, which is concluded as follows. 1. A range of factor are tested to conclude that the larger factor applied is, the longer the project duration is, whereas less amount of project buffers would be inserted. 2. The simulation is conducted based on the parameter set in CCPM+, and SSQ demonstrates shorter time of project compared with the method of $50 \%$ fixed length. It is generally more optimistic to size the project buffer when the SSQ method is adopted.

In terms of the methodological approach, this paper adopts literature review and computerized simulation on simple examples. To better demonstrate the conclusion made, further study based on practical project could be researched and tested.

\section{Reference}

[1]Goldratt, E.M., (1997). Critical Chain. New York: The North River Press.

[2] Advanced Projects, (2008). Help in CCPM+ software

[3] Pidd M. (1988.) Computer simulation in management science. 4th ed. Chichester : John Wiley

[4]Schieman N.(2002) “CCPM and Project Success” MSc. Dissertation. University of Maastricht.

[5]Tukel et al, (2006). An investigation of buffer sizing techniques in critical chain scheduling. European Journal of Operational Research 172, 401-416 